\title{
Active Giant Peripheral Osteoma of the Mandible. Presentation of one case with follow-up to 6 years.
}

\author{
Alejandro Donohué-Cornejo ${ }^{1}$, Laura Elena Franco Garrocho ${ }^{2}$, Surisadey Albarrán-Vergara ${ }^{3}$, Luis Alberto \\ Gaitán Cepeda $^{3}$ \\ ${ }^{1}$ Departamento de Estomatología. Instituto de Ciencias Biomédicas. Universidad Autónoma de Ciudad Juárez. \\ ${ }^{2}$ Clínica de Cirugía Maxilofacial. Universidad Autónoma de Ciudad Juárez \\ ${ }^{3}$ Laboratorio de Patología Bucal Clínica y Experimental. División de Estudios de Posgrado e Investigación. Facultad de Odonto- \\ logía. Universidad Nacional Autónoma de México.
}

Correspondence:

Laboratorio de Patología Bucal Clínica y Experimental.

División de Estudios de Posgrado e Investigación. Facultad de Odontología. Universidad Nacional Autónoma de México.

Circuito de la Investigación s/n, Ciudad Universitaria,

Coyoacán 04510 D.F

Mexico

e-mail:lgaitan@servidor.unam.mx

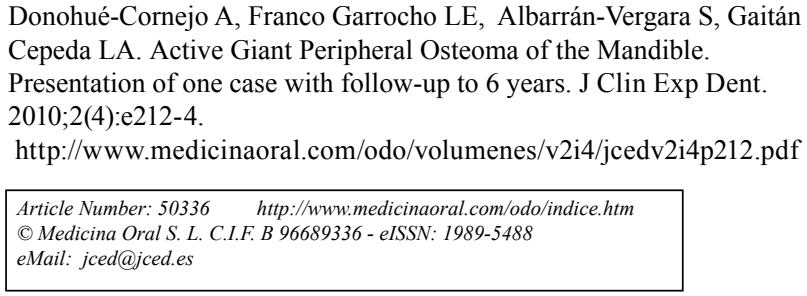

\begin{abstract}
Osteoma, a benign neoplasia of bone tissue, is considered a quiescent neoplasm. An active osteoma is an uncommon lesion. We presented a case of a mandibular osteoma with an unusual active behavior. In January of 2001 was admitted in the Oral Medicine Service a 11-years old female because an asymptomatic growth in the left side of their face with two years of evolution. Radiographical examination revealed a well circumscribed spherical radiopaque lesion of approximately $2 \times 2 \mathrm{~cm}$ in diameter on lateral side of the left angle of the mandible. Microscopically the surgical sample coming from incisional biopsy showed normal bone tissue. A diagnosis of osteoid osteoma was emitted. Surgical treatment was indicated and refused by the parents of the patient. In August 2007 the same was readmitted because an increase in their facial asymmetry. Radiographical examination revealed an increase in the size of the radiopaque area to $3 \times 3 \mathrm{~cm}$. A $99 \mathrm{~m}$ Tc MDP bone scintigraphy was indicated and performed. Because the scintigraphy showed bone activity the surgical elimination of the whole lesion was indicated and done. Microscopically the lesion was composed by vital mature lamellar and trabecular bone. A diagnosis of giant active osteoma of the mandible was emitted. The maxillofacial surgeon should awake by the possibility of an osteoma shows neoplastic activity.
\end{abstract}

Key words: Osteoma, mandible, peripheral, bone scintigraphy. 


\section{Introduction}

The most frequent localization of the osteoma, a benign neoplasm of bone tissue, in the head is paranasal sinuses, mastoid, middle ear and jaws. Osteoma occurs mainly in the second and fourth decade of life, being more common in males (1). Because by itself is asymptomatic the osteoma of the maxillaries usually is diagnosed in a routinely radiographical exam. Radiographically the osteoma is characterized by a single or multiple well-circumscribed radiopaque areas $(1,2)$. Their symptomatology is in accordance to their topography, e.g. sinusitis, localized pain, headache, nasal obstruction, exophthalmos, facial asymmetry, difficulty to opening the mouth and important to the present report, facial asimetry $(1,2)$. Osteoma is considered as a low growth neoplasm or even more, like an inactive neoplasm. The active giant osteomas of the mandible are rare lesions. With the objective of contribute to a better knowledge of this uncommon behavior of this neoplasm, we present one case of active giant peripheral osteoma in the mandible of a teenager with follow-up of 6 years.

\section{Case report.}

In 2001 a 11 years old girl presented in the Oral Medicine Clinic at Department of Stomatology, Biomedical Science Institute, Autonomous University of Ciudad Juárez, México because an asymptomatic swelling involving the left side of her face. The extra and intraoral examination revealed a swelling in the left angle of the mandible. The patient and their parents not reveal antecedents of facial trauma. Radiographical examination showed a single well-circumscribed radiopaque area in the lateral side of the left angle of the mandible. The radiopaque area sized $2 \times 2 \mathrm{~cm}$ approximately (Fig.1).

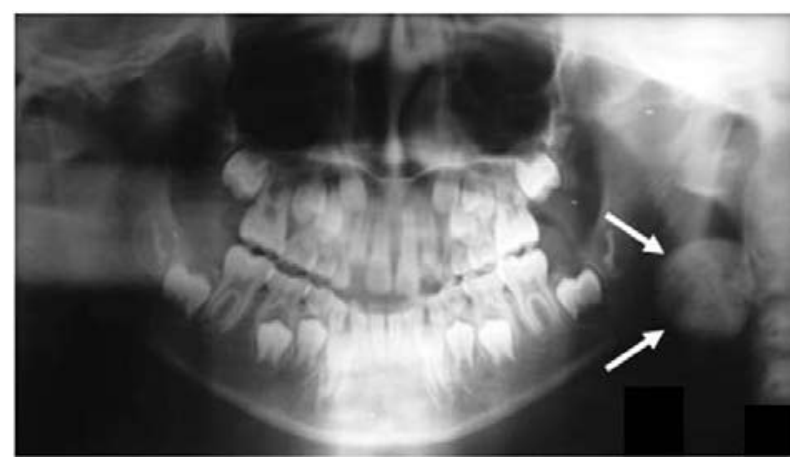

Fig 1. Radiographical characteristics of one case of active giant osteoma. Orthopantomography taken in 2001. Observes the spherical well circumscribed radiopaque area (white arrows) located at the lateral side of the left angle of the mandible with approximately $2 \times 2$ $\mathrm{cm}$ in diameter.

With the presumptive clinical and radiographical diagnosis of osteoma, the patient was referred to the Oral and Maxillofacial Surgery clinic at same institution for incisional biopsy. The surgical sample was fixed at $10 \%$ buffered formalin by 48 hours. After decalcification in $6 \%$ nitric acid for 24 hours the sample was embedded in paraffin, cut into $5 \mu$ and stained with hematoxylin and eosin technique. Microscopically vital and mature lamellar bone was observed. A diagnosis of osteoid osteoma was emitted. Surgical treatment was indicated but refused by the parents of the patient. A close follow-up of the patient was proposed, however after 6 months of follow-up the patient lose their control examination and eventually leave it.

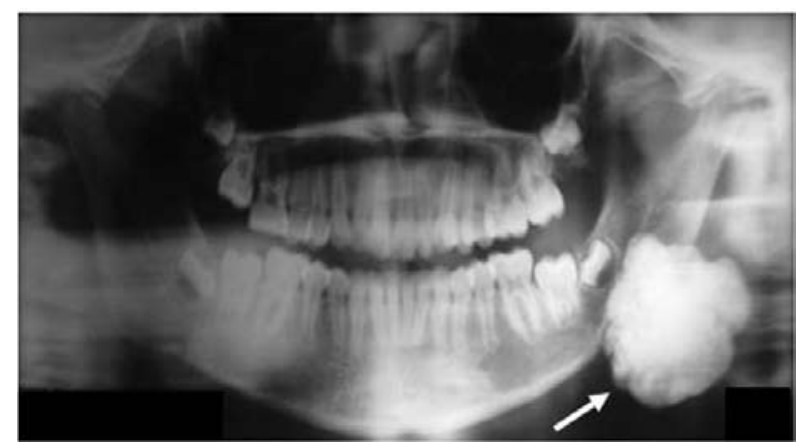

Fig 2. Radiographical characteristics of one case of active giant osteoma. Orthopantomography taken in 2007. Observes the radiopaque area located on the lateral side of the left ascendant ramus of the mandible. The lesion growths towards the cervical soft tissues (white arrow).

In 2007 the patient comes back her she was awake of an increase of the swallow on the left side of her face. The extraoral examination revealed a great facial asymmetry due to the presence of a hard, not-mobile, asymptomatic swallow of the lateral side of the left angle and ramus of the mandible. The skin covered the swallow was normal in color. Radiographical examination showed a single radiopaque area of approximately $3 \times 3 \mathrm{~cm}$ located on the left angle and ramus of the mandible involving the soft tissues of the neck (Fig. 2). To asses bone activity a 99m Tc MDP scintigraphy was indicated and performed. The scintigraphy showed bone activity in the region lateral of the left mandibular (Fig. 3) corresponding to the radiopaque area. Due to these findings a presumptive diagnosis of active osteoma was emitted and indicated the total remove of the tumor. The patient was referred to the Maxillofacial Surgery Service to exeresis of the whole lesion. The sample was fixed at $10 \%$ buffered formalin by 48 hours. Macroscopically the specimen ( $4.2 \mathrm{x}$ $3.7 \times 3.5 \mathrm{~cm}$ ) was composed by multiple brownish fragments of soft and hard tissue. The largest fragment had a smooth surface with a spherical shape. After decalcification in $6 \%$ nitric acid, representative fragments of the sample were embedded in paraffin, cut at $5 \mu$ and stained with hematoxylin and eosin technique. Microscopically the lesion was composed by vital mature lamellar and trabecular bone and by normal bone marrow. The entire lesion was surrounding by a fibro-osseous capsule. Additionally were identifies striated muscle tissue; fat 


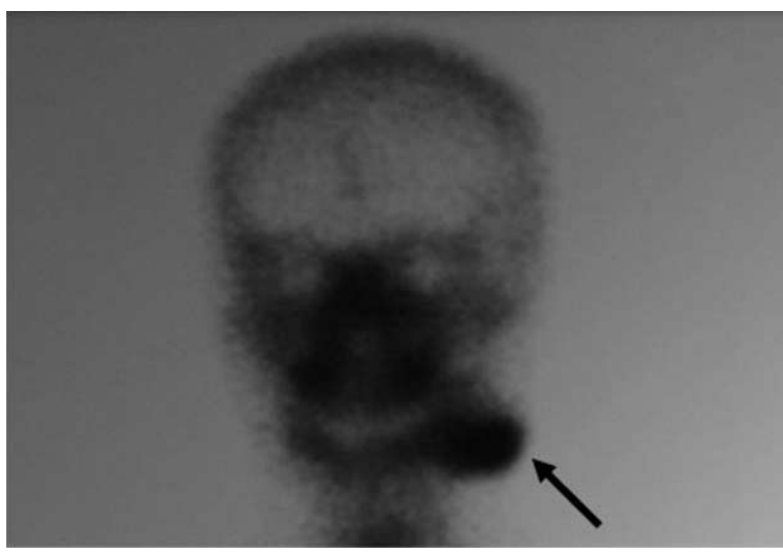

Fig 3. Imagenological characteristics of one case of active giant osteoma. This Tc-99m MDP scintigraphy shows activity in the lateral side of the left angle and ascendant ramus of the mandible (black arrow). Notice the coincident area with figure $B$.

tissue and nerve fibers. A diagnosis of active giant osteoma of the mandible was emitted.

\section{Discussion}

We present a case of active giant peripheral osteoma in the mandible of an 11-years girl. Our case agrees with the topography reported but disagrees in regard to gender predilection (2). The osteoma is originated at the endosteum (central osteoma) or at the periosteum (peripheral osteoma). The peripheral osteoma show a centrifugal/ centripetally growth $(2,3)$. The more frequent type of osteoma are the peripheral ones being their most common site in the skull the lateral angle of the mandible (1-4). It has been proposed that osteoma could be in consequence of infection or trauma (4-6). The medical file of the patient not showed antecedent of trauma on facial bones, specifically the mandible.

To our knowledge this is the first report of one active giant osteoma of the mandible with neoplasic activity demonstrated by scintigraphy. The osteoma is considered as a slow growth or quiescent neoplasm (1-4). In such a way frequently is recommended periodical (3-6 months) observation, however the success of this recommendation depends of the adherence to the followup of the patients $(1,5,6)$. If the osteoma has neoplasic activity is strongly recommended their surgical excision. The present case shows the possibility that a quiescent neoplasma shows an active growth in accordance to their neoplasic nature. The osteoma may growth toward soft tissue, with systemic or local complications. In the present case, the osteoma growths toward the cervical soft tissue and in consequence the surgical treatment was most invasive increase the possible complications. Maxillofacial surgeon should be alert to identify this type of lesions and to emphasize their total excision.

\section{References}

1. Sayan NB, Uçok C, Karasu HA, Günhan O. Peripheral osteoma of the oral and maxillofacial region: A study of 35 new cases. J Oral Maxillofac Surg. 2002;60: 1299-301.

2. Larrea-Oyarbide N, Valmaseda-Castellón E, Berini-Aytés L, GayEscoda C. Osteomas of the craniofacial region. Review of 106 cases. J Oral Pathol Med. 2008;37: 38-42.

3. Woldenberg Y, Nash M, Bodner L. Peripheral osteoma of the maxillofacial region. Diagnosis and management: a study of 14 cases. Med Oral Patol Oral Cir Bucal. 2005;10:E139-42.

4. Kaplan I, Calderon S, Buchner A. Peripheral osteoma of the mandible: a study of 10 new cases and analysis of the literature. J Oral Maxillofac Surg. 1994: 52: 467-70.

5. Johann AC, de Freitas JB, de Aquiar MC, de Araújo NS, Mesquita RA. Peripheral osteoma of the mandible: case report and review of the literature. J Craniomaxillofac Surg. 2005;33: 276- 81.

6. Kashima K, Rahman OI, Sakoda S, Shiba R. Unusual peripheral osteoma of the mandible: report of 2 cases. J Oral Maxillofac Surg. 2000;58: 911-3. 